\title{
Psychological Distress Among HIV Healthcare Providers During the COVID-19 Pandemic in China: Mediating Roles of Institutional Support and Resilience
}

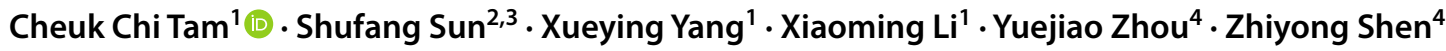

Accepted: 14 October 2020 / Published online: 21 October 2020

(c) Springer Science+Business Media, LLC, part of Springer Nature 2020

\begin{abstract}
Psychological distress among healthcare providers is concerning during COVID-19 pandemic due to extreme stress at healthcare facilities, including HIV clinics in China. The socioecological model suggests that psychological distress could be influenced by multi-level factors. However, limited COVID-19 research examined the mechanisms of psychological distress among HIV healthcare providers. This study examined organizational and intrapersonal factors contributing to psychological health during COVID-19 pandemic. Data were collected via online anonymous surveys from 1029 HIV healthcare providers in Guangxi, China during April-May 2020. Path analysis was utilized to test a mediation model among COVID-19 stressors, institutional support, resilience, and psychological distress (PHQ-4). Thirty-eight percent of the providers experienced psychological distress (PHQ-4 score > 3). Institutional support and resilience mediated the relationship between COVID-19 stressors and psychological distress. Psychological distress was common among Chinese HIV healthcare providers during COVID-19 pandemic. Psychological health intervention should attend to institutional support and resilience.
\end{abstract}

Keywords COVID-19 $\cdot$ HIV $\cdot$ Healthcare providers $\cdot$ Psychological distress $\cdot$ Institutional support $\cdot$ Resilience

\section{Introduction}

\section{Psychological Distress Among Healthcare Providers During the COVID-19 Pandemic}

Since the first case emerged in December 2019 in China, the novel coronavirus disease 2019 (COVID-19) has spread rapidly across the world and become a worldwide public

Cheuk Chi Tam

ctam@mailbox.sc.edu

1 South Carolina SmartState Center for Healthcare Quality, Department of Health Promotion, Education, and Behavior, Arnold School of Public Health, University of South Carolina, Discovery I, Suite 408, 915 Greene Street, Columbia, SC 29208, USA

2 Department of Behavioral and Social Science, Brown University School of Public Health, Providence, RI, USA

3 Department of Psychiatry and Human Behavior, Brown University Alpert Medical School, Providence, RI, USA

4 Guangxi Center for Disease Control and Prevention, Nanning, Guangxi Zhuang Autonomous Region, People's Republic of China health emergency [1]. The COVID-19 pandemic has led to numerous detrimental consequences, including fatalities and significant socio-economic impacts (e.g., significant medical costs, increased unemployment and financial stress) [2, 3]. In addition to medical and economic consequences, growing literature suggests prevalent psychological distress (e.g., depression and anxiety) during the COVID-19 pandemic [4-6]. Healthcare providers may be particularly vulnerable to psychological distress due to a variety of factors. Relative to non-medical workers, healthcare providers encounter greater COVID-19 threats (e.g., the high risk of infectious exposure, shortage of personal protection equipment, and increasing workloads) detrimental to their psychological health $[7,8]$. A recent meta-analysis on 10 COVID-19 studies has identified a high prevalence of psychological distress among healthcare providers (23.8\% depression, 22.8\% anxiety) [9]. Psychological distress in healthcare providers during COVID-19 have been associated with physical problems, including insomnia and somatic symptoms [10,11]. However, existing COVID-19 studies regarding psychological distress among healthcare providers mostly focused on nurses or surgical staffs $[12,13]$, and limited research have focused on other providers. 


\section{COVID-19 Stressors Among HIV Healthcare Providers}

Psychological distress among HIV healthcare providers in China could be particularly concerning due to several COVID-19 related stressors at HIV clinics. First, as the first country to experience this novel infectious disease, the Chinese medical system lacked initial preparedness for the immediate transitions necessary at HIV healthcare services (e.g., in-person to Internet-based medical care) [14]. Second, medical management system in China is relatively centralized, and HIV patients usually receive HIV care at designated clinics. Thus, city lockdowns and quarantine enforcement during the pandemic could lead to massive HIV care disruptions $[15,16]$, which may adversely affect providers' psychological health. Third, due to HIV providers' background in infectious disease and a shortage of providers on the frontline, many HIV healthcare providers were assigned to provide COVID-19 care and control [15], leading to increased workload. However, despite multiple stressful burdens at HIV clinics during the COVID-19 pandemic, no studies have yet attended to the influences of these stressors on psychological distress in Chinese HIV healthcare providers.

\section{An Ecological Perspective of Psychological Distress Among HIV Healthcare Providers}

From an ecological perspective, the psychological impact of COVID-19 stress among HIV healthcare providers may be shaped by factors from different levels. The ecological systems theory posits that individuals' health outcomes are determined by factors of multi-level systems, such as microsystem (e.g., intrapersonal), exosystem (e.g., organizational), and macrosystem (e.g., contextual) [17]. In addition to highlighting systematic structure of factors, this theory illustrates the underlying mechanism of multi-level factors on individual's psychological outcomes, suggesting that factors from distal levels (e.g., contextual factors) can indirectly impact psychological outcomes through affecting factors from proximal levels (e.g., organizational and intrapersonal) [18]. In other words, distal factors would first affect proximal factors, and, in turn result in psychological consequences for the individual. Thus, COVID-19 stressors as a contextual factor may indirectly affect psychological distress through organizational and intrapersonal factors among Chinese HIV healthcare providers. To better understand the stress-psychological-distress mechanism among Chinese HIV healthcare providers, it is worth identifying organizational and intrapersonal factors contributing to psychological health in the COVID-19 context.

\section{COVID-19 Stressors, Institutional Support, Resilience, and Psychological Distress}

Institutional support has been consistently identified as an organizational factor that influences psychological distress in healthcare providers. Institutional support can be understood as supportive responses offered by institutes for addressing physical, emotional, and psychosocial needs of the workforce $[19,20]$. Systematic reviews of literature among healthcare providers documented that institutional support (e.g., organizational climates and infrastructure of health facilitates) was associated with psychological health $[21,22]$. In addition to the direct effect, as suggested in the ecological systems theory, previous studies identified an indirect effect of institutional support, such that individuals with a lower occupational stress perceived more institutional support, and, in turn, individuals reported a lower psychological distress [23, 24]. It is particularly worth noting that institutes play a critical role in providing support for healthcare providers during other similar public health crisis (e.g., influenza pandemic) [25]. In the context of COVID-19, a focus group among 69 frontline healthcare providers during the outbreak highlighted the lack of institutional supportive responses to COVID-19 as a direct source of distress (e.g., 'uncertainty if their organization would take care of their personal and family needs if they become infected by COVID-19'), suggesting an importance of institutional support in this unprecedented public health emergency [19]. Taken all together, institutional support (i.e., institutional supportive responses to COVID-19) could be an important organizational-level factor to directly and indirectly affect psychological distress among HIV healthcare providers in face of COVID-19 stressors.

As an intrapersonal factor, psychological resilience (or resilience) refers to personal abilities and resources (e.g., optimism, tenacity, tolerance to stress, and self-efficacy) and is a protective factor for coping with or overcoming stressful circumstances $[26,27]$. For instance, a meta-analysis on 60 studies found resilience to be a protective factor for psychological distress across diverse samples [28]. Similar to institutional support, resilience could have an indirect effect in the association between pandemic stressors and psychological distress [29-31]. Specific to the pandemic context, a recent study found a negative association of resilience with psychological distress among Chinese healthcare providers during the COVID-19 outbreak [32].

Further, as a higher-level factor (exosystem), institutional support could affect resilience (at microsystem), and, in turn, influence psychological outcomes. Indeed, a previous study have shown an indirect effect of resilience in the relationship between institutional support and depression among individuals with high occupational stress (e.g., frontline 
correctional officers) [33]. In line with the institutional support influences on resilience, COVID-19 stressors may exert influences on psychological distress by the intermediating effects of institutional support and resilience. That is, the stress-psychological distress mechanism among HIV healthcare providers would occur through a serial association, including a link between COVID-19 stressors and institutional support and a link between institutional support and resilience, followed by a link between resilience and psychological distress. Understanding this mechanism among HIV healthcare providers in China is important, as it can potentially guide timely and needed interventions and contribute to scientific knowledge on the utility of an ecological perspective in psychological health during extreme stress.

\section{The Present Study}

The aim of the present study was to investigate the underlying mechanisms among COVID-19 stressors, institutional support, resilience, and psychological distress among HIV healthcare providers in China. Figure 1 depicts our research hypotheses in the form of a serial indirect model, guided by the ecological systems theory [17], Specifically, we hypothesized that (1) COVID-19 stressors would be positively associated with psychological distress; (2) institutional support would mediate the association between COVID-19 stressors and psychological distress; (3) resilience would mediate the association between COVID-19 stressors and psychological distress; (4) the association between COVID-19 stressors and psychological distress would be serially mediated by institutional support and resilience.

\section{Method}

\section{Participants and Procedure}

The present cross-sectional study used data collected by a online survey from a convenience sample of healthcare providers who engaged in HIV care services in Guangxi, one of the Chinese regions with the fastest growth of the HIV epidemic. Since 2014, Guangxi reported the third highest number of HIV cases among China's 31 provinces [34, 35]. Data were collected from April 2020 through May 2020, a time when the confirmed COVID-19 cases exceed 82,000 in China according to Chinese Center of Disease Control and Prevention (CDC) [36]. The SO JUMP system technology, a popular Chinese online survey platform [37], was used for data collection. Eligibility criteria for participants included: (1) currently providing HIV-related care and services in Guangxi; and (2) being 18 years of age or older.

Personnel in Guangxi CDC contacted HIV healthcare providers across the region by an email invitation to the 15 -min online survey. A total of 1280 HIV healthcare providers from 14 counties (see Table 1) in Guangxi responded the invitations and participated in the survey. Participants were provided with an electronic consent prior to the survey started. The consent showed information regarding study purpose, voluntary nature, and confidentiality. After obtaining consent, the SO JUMP system navigated participants to the online survey. Data were saved at the SO JUMP system with the password protection. Participants were encouraged to share the survey invitation with their colleagues. The research protocol was approved by the Institutional Review Boards at both University of South Carolina in the United States and Guangxi CDC in China.

\section{Measures}

\section{Demographics}

Participants provided their demographic (i.e., age, gender, residence, educational level, and marital status) and occupational information including professional position (i.e., nurse, lab personnel, CDC staff, physician, and other), professional ranking (i.e., no ranking, entry level, middle level, senior level, and advanced level), administrative ranking (i.e., no ranking, department leader, hospital/CDC director, and other), and level of affiliated institution (i.e., province, city, country, and rural).
Fig. 1 Hypothesized conceptual model among COVID-19 stressors, institute social support, resilience, and psychological distress among HIV healthcare providers

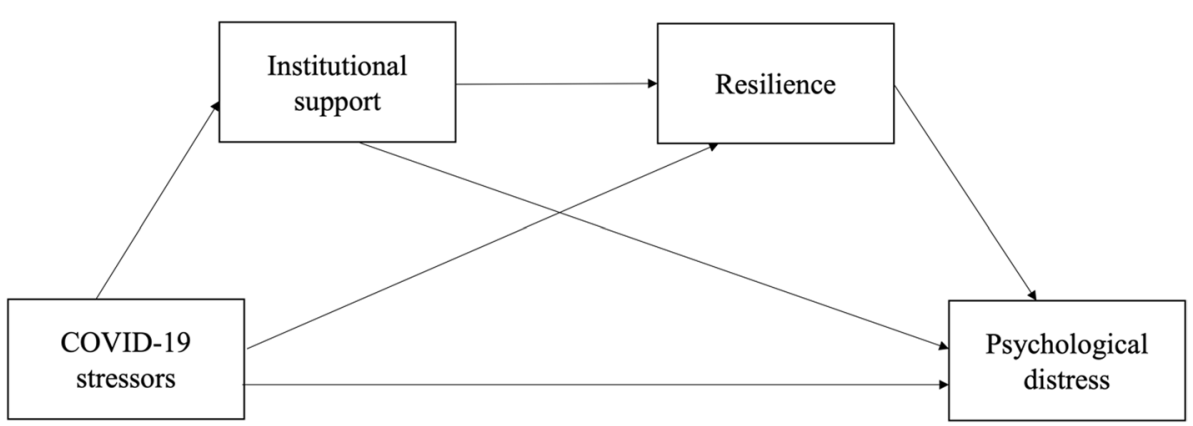


Table 1 Demographic characteristics and psychological distress among HIV healthcare providers $(N=1029)$

\begin{tabular}{|c|c|}
\hline & $n(\%)$ \\
\hline Age, Mean (SD) & $38.39(9.20)$ \\
\hline \multicolumn{2}{|l|}{ Gender } \\
\hline Male & $395(38.4 \%)$ \\
\hline Female & $634(61.6 \%)$ \\
\hline \multicolumn{2}{|l|}{ Marital status } \\
\hline Unmarried & $145(14.1 \%)$ \\
\hline Umarried cohabitating & $14(1.4 \%)$ \\
\hline Married/remarried & $840(81.6 \%)$ \\
\hline Separated & $2(0.2 \%)$ \\
\hline Divorced & $23(2.2 \%)$ \\
\hline Widowed & $5(0.5 \%)$ \\
\hline \multicolumn{2}{|l|}{ Affiliated institute level } \\
\hline Province level & $11(1.1 \%)$ \\
\hline City level & $159(15.5 \%)$ \\
\hline County level & $326(31.7 \%)$ \\
\hline Rural level & $533(51.8 \%)$ \\
\hline \multicolumn{2}{|l|}{ Education level } \\
\hline Middle school & $111(10.8 \%)$ \\
\hline High school & $75(7.3 \%)$ \\
\hline College diploma & $491(47.7 \%)$ \\
\hline Bachelor's degree & $343(33.3 \%)$ \\
\hline Master's degree & $9(0.9 \%)$ \\
\hline \multicolumn{2}{|l|}{ Professional position } \\
\hline Nurse & $155(15.1 \%)$ \\
\hline Lab personnel & $36(3.5 \%)$ \\
\hline Local CDC staff & $387(37.6 \%)$ \\
\hline Physician & $219(21.3 \%)$ \\
\hline Other & $232(22.5 \%)$ \\
\hline \multicolumn{2}{|l|}{ Residence } \\
\hline Baise & $64(6.2 \%)$ \\
\hline Beihai & $43(4.2 \%)$ \\
\hline Fangchenggang & $33(3.2 \%)$ \\
\hline Guigang & $52(5.1 \%)$ \\
\hline Guilin & $98(9.5 \%)$ \\
\hline Hechi & $48(4.7 \%)$ \\
\hline Hezhou & $46(4.5 \%)$ \\
\hline Laibin & $44(4.3 \%)$ \\
\hline Liuzhou & $64(6.2 \%)$ \\
\hline Nanning & $304(29.5 \%)$ \\
\hline Qinzhou & $71(6.9 \%)$ \\
\hline Songzuo & $46(4.5 \%)$ \\
\hline Wuzhou & $20(1.9 \%)$ \\
\hline Yulin & $82(8.0 \%)$ \\
\hline PHQ-4 - sum score ${ }^{\mathrm{a}}$ & $2.08(2.10)$ \\
\hline Normal & $626(60.8 \%)$ \\
\hline Mild & $344(33.4 \%)$ \\
\hline Moderate & $56(5.4)$ \\
\hline Severe & $3(0.3 \%)$ \\
\hline
\end{tabular}

${ }^{\mathrm{a}}$ Categories were determined using the diagnosis guideline $(0-2$, normal; 3-5, mild; 6-8, moderate; 9-12 severe) suggested by Kroenke \& Spitzer, Williams, \& Lowe (2009)

$S D$ standard deviation

\section{Psychological Distress}

Psychological distress was measured through the Patient Health Questionnaire-4 (PHQ-4; Chinese version) [38, 39]. The PHQ-4 had four items asking mood disorder symptoms (two items for depression and other two items for anxiety) in the past two weeks. All items were rated on a four-point scale ranging from 0 (not at all) to 3 (nearly every day). Given that the depressive and anxiety symptoms were highly correlated $(r=0.71)$, a sum score of all four items was used to represent psychological distress, with higher scores indicating higher levels of psychological distress. In addition, we divided responses into four psychological distress levels (i.e., normal [0-2], mild [3-5], moderate [6-8], and serve [9-12]) according to the diagnosis guideline suggested by Kroneke et al. [38]. The Cronbach's alpha of the PHQ-4 was 0.86 in the current study.

\section{COVID-19 Stressors}

A self-developed and 20-item checklist was used to evaluate the COVID-19 stressors. Participants were asked to answer $(0=$ 'No'; $1=$ 'Yes' $)$ whether 20 potential stressful events occurred at the hospital or HIV clinics during the COVID-19 outbreak, such as care of COVID-19 patients (e.g., "Treated/ provided care for patients infected by COVID-19"), occupational overload (e.g., "Hospital was overloaded due to the COVID-19 outbreak"), difficulties in the delivery of healthcare (e.g., "Inadequate personal equipment for COVID-19 prevention"), and daily life disturbance (e.g., "Guilt because I was unable to take care of my family during the outbreak"). A sum score was calculated, with a higher score representing the exposure to more COVID-19 stressors. Cronbach's alpha was 0.78 in the current sample.

\section{Institutional Support}

Institutional support was measured through a self-developed scale with five items describing supportive responses (i.e., financial, emotional, medical, and technical) from participants' institutes to cope with difficulties at clinics due to COVID-19 (e.g., "My institute offers trainings about the provision of HIV care during the COVID-19 outbreak" and "My institutes provide sufficient personal preventive equipment"). Participants rated items on a four-option scale ( $1=$ strongly disagree to $4=$ strongly agree). A sum score was generated, with higher scores presenting higher levels of institutional support. Cronbach's alpha of this scale was 0.82 .

\section{Resilience}

Psychological resilience among HIV healthcare providers was assessed using a measure adapted from the 
Connor-Davidson resilience scale (CD-RISD) [40]. A total of nine items assessed personal capacities in response to stress such as tenacity, tolerance of negative affect, positive acceptance of change, self-efficacy to deal with stress, and positive view of adversities (e.g., "able to adapt to change" and "can deal with whatever comes"). Participants were asked to rate items from 1 (not at all) to 5 (nearly all the time). A higher sum score indicated a greater level of resilience. Internal reliability for the current study sample was excellent $(\alpha=0.93)$.

\section{Data Analysis}

First, data were screened for proper coding and random or careless responses. Mahalanobis distance $\left(D^{2}\right)$ [41] was utilized to identify multivariate outliers. Considering that path analysis model can be strongly jeopardized by multivariate outliers [42], we followed the suggested best practice and removed 251 surveys with multivariate outliers and random responses, resulting in an analytical sample of 1029 participants. Kurtosis and skewness tests were used to detect normality of data. Descriptive statistics were performed on demographic variables and psychological distress. Bivariate analyses, Pearson's correlation coefficients, were used to assess correlations among study variables (COVID-19 stressors, institute support, resilience, and psychological distress). Pearson's (for continuous variables) and Point-biserial correlation tests (for categorical variables) were used to examine the bivariate relationship of demographic variables and psychological distress. Descriptive statistics and bivariate analyses were employed using SPSS software version 26.

Path analysis was employed to examine the hypothesized model (Fig. 1). This model adjusted for significant demographic variables in bivariate analyses. All study variables were entered as manifest factors and the standardized regression weights for all paths between study variables were calculated. Given that endogenous variables (psychological distress, institutional social support, and resilience) were continuous and normally distributed (Kurtosis and Skewness estimates closed to 1) [43], the path analysis model was tested using maximum likelihood (ML) estimation as suggested by Meyers et al. [41]. Model's goodness of fit was determined by several indices, including the root mean square of approximation (RMSEA), the comparative fit of index (CFI), the Tucker-Lewis index (TLI), and the standardized root mean squared residual (SMSR) [41]. According to $\mathrm{Hu}$ an Bentler [44], the suggested cutoff values were 0.95 for CFI, 0.95 for TLI, 0.05 for RMSEA, and 0.06 for SMSR, with higher CFI and TLI and lower RMSEA and SMSR indicating a greater fit.

The indirect effects of institute support and resilience in the relationship between COVID-19 stressors and psychological distress were examined using the delta $\mathrm{z}$ score [45].
The indirect effect analysis examined whether institutional support and resilience partially or completely mediated the relationship between COVID-19 stressors and psychological distress. As suggested by Baron and Kenny [46], complete mediation is identified when a mediation factor reduces the regression coefficients between independent variable (COVID-19 stressors) and criterion variable (psychological distress) to zero (non-significant). Partial mediation occurs when a mediation factor reduces the regression coefficient between independent and criterion variables, but the regression coefficient remains significant. Path analysis was performed in Mplus version 8 [45].

\section{Results}

\section{Demographic Characteristics and Descriptive Statistics}

As shown in Table 1, participants were 38.39 years $(S D=9.20)$ of age on average. The sample was mostly female [634 (61.6\%)] and married [840 (81.6\%)]. Fortyseven percent of HIV healthcare providers $(n=491)$ received a college diploma. Twenty-nine percent of participants $(n=304)$ were from Nanning. In terms of occupational characteristics, the majority of sample reported working in an institute at the rural level [533 (51.8\%)] or county level [326 (31.7\%)]. Most healthcare providers were self-identified as local CDC staffs [387 (37.6\%)], followed by physicians [219 (21.3\%)], nurses [155 (15.1\%)], and lab personnel [36 (3.5\%)].

In terms of psychological distress, the sum score of the PHQ-4 scale was 2.08 on average $(S D=2.10$, Range $=[0,9])$. Using the psychological distress level diagnosis guideline suggested by Kroneke et al. (2009), 33.4\% of participants experienced mild level of distress, $5.4 \%$ reported moderate distress, and $0.3 \%$ had severe distress.

\section{Bivariate Analysis}

As presented in Table 2, Pearson's correlation tests suggested that COVID-19 stressors was positively correlated

Table 2 Correlations among COVID-19 stressors, resilience, institute support, and psychological distress $(N=1029)$

\begin{tabular}{lllll}
\hline & 1 & 2 & 3 & 4 \\
\hline 1. COVID-19 stressors & 1 & & & \\
2. Resilience & $-0.13^{* * * *}$ & 1 & & \\
3. Institutional support & $-0.11^{* * * *}$ & $0.28^{* * * *}$ & 1 & \\
4. Psychological distress & $0.33^{* * *}$ & $-0.43^{* * *}$ & $-0.21^{* * *}$ & 1 \\
\hline
\end{tabular}

$* p<.05 ; * * p<.01 ; * * * p<.001$ 
with psychological distress $(r=0.33, p<0.001)$ and negatively correlated with resilience $(r=-0.13, p<0.001)$ and institutional support $(r=-0.11, p<0.001)$. Psychological distress was negatively correlated with resilience $(r=-0.43, p<0.001)$ and institutional support $(r=-0.21, p<0.001)$. Resilience was positively correlated with institutional support $(r=0.28, p<0.001)$.

Pearson's and Point-biserial correlation tests suggested that psychological distress was significantly correlated with age $(r=-0.11, p=0.001)$, gender $(r=0.09$, $p=0.006)$, marital status $(r=-0.16, p<0.001)$, and affiliated institute level $(r=0.12, p<0.001)$. Professional position was not significantly correlated with psychological distress $(r=0.02, p=0.465)$.

\section{Path Analysis}

The final path analysis model with standardized regression coefficients is shown in Fig. 2. According to bivariate results, this model controlled for age, gender, marital status, and affiliated institute level. The Chi-square test of the model (Chi-square $=11.06, d f=7, p=0.136$ ) and the model-fit indices $(\mathrm{CFI}=0.99$; $\mathrm{TLI}=0.98, \mathrm{RMSEA}=0.02$, and SRMR $=0.02$ ) suggested an excellent fit to data. COVID-19 stressors were positively associated with psychological distress $(\beta=0.27, p<0.001)$. The paths from COVID-19 stressors to institutional support and resilience were negative $(\beta=-0.10, p=0.001 ; \beta=-0.10$, $p=0.001$; respectively). Institutional support and resilience were negatively associated with psychological distress $(\beta=-0.07, p=0.01 ; \beta=-0.36, p<0.001$; respectively). Institutional support was positively associated with resilience $(\beta=0.27, p<0.001)$. The model explained $28.5 \%$ of variance in psychological distress, $12.4 \%$ of variance in resilience, and $2.4 \%$ of variance in institutional support.

\section{Indirect Effects}

The indirect effects in the hypothesized model were presented in Table 3. As hypothesized, COVID-19 stressors had a direct effect on psychological distress $(\beta=0.27$, delta $z=9.24, p<0.001,95 \%$ confidence interval $[C I] 0.22,0.31)$. Results suggested significant indirect effects $(\beta=0.05$, $p<0.001,95 \%$ CI $0.03,0.07$ ) in the association between COVID-19 stressors and psychological distress by resilience and institutional support were significant $(\beta=0.03$, delta $z=3.08, p<0.001,95 \%$ CI 0.02, $0.05 ; \beta=0.01$, delta $z=1.97, p=0.042,95 \%$ CI $0.002,0.01$; respectively). In addition, the total indirect effect of institutional support together with resilience was statistically significant $(\beta=0.01$, delta $z=3.12, p=0.003,95 \% C I 0.01,0.02$ ). These results suggested partial mediations of institutional support and resilience (solely or jointly) in the association between COVID-19 stressors and psychological distress.

In the relationship between institutional support and psychological distress, results suggested a direct effect of institutional support $(\beta=-0.07$, delta $z=-2.54$, $p=0.011,95 \% C I-0.12,-0.03)$ and an indirect effect of resilience $(\beta=-0.10$, delta $z=-7.43, p<0.001,95 \%$ $C I-0.12,-0.08)$, indicating that resilience partially mediated the association between institutional support and psychological distress.

\section{Discussion}

Guided by an ecological perspective, the current study explored psychological distress among Chinese HIV healthcare providers and examined the roles of COVID-19 stressors, institutional support, and resilience in psychological distress. To the best of our knowledge, this is the first attempt to document psychological distress and apply an ecological framework for examining the effects of protective factors on
Fig. 2 Path analysis on a serial mediation model among COVID-19 stressors, institutional support, resilience, and psychological distress among HIV healthcare providers

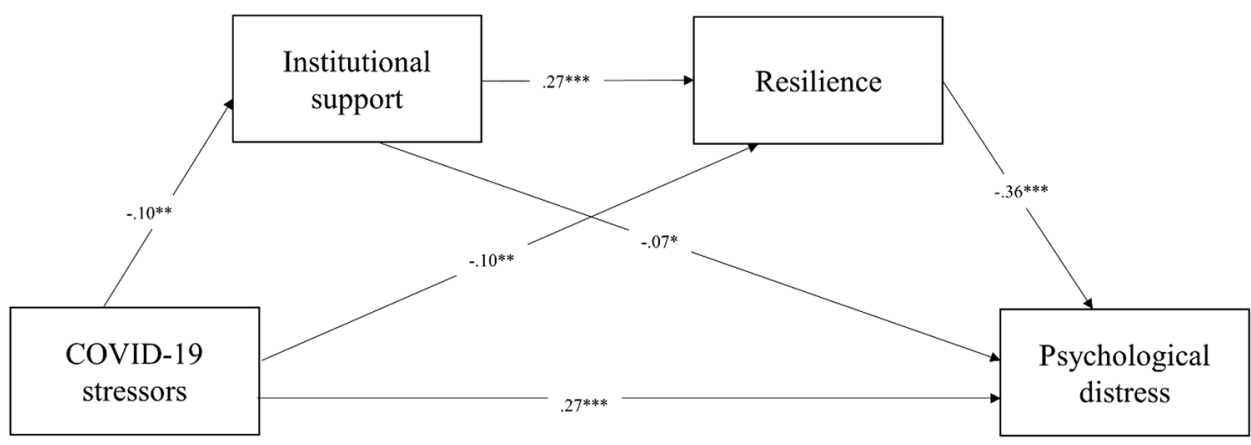

Model-fit indices: Chi-square $=11.06, d f=7, p=.136 ; \mathrm{CFI}=.99 ; \mathrm{TLI}=.98 ; \mathrm{RMSEA}=.02 ; \mathrm{SRMR}=.02$

Controls: age, gender, martial status, and affiliated institute level

Explained variances: Psychological distress (28.5\%), resilience $(12.4 \%)$, institutional support $(2.4 \%)$ 
Table 3 Indirect effect analyses for hypothesized model

\begin{tabular}{|c|c|c|c|c|}
\hline Effects & $B$ & S.E & p-value & $95 \% C I$ \\
\hline \multicolumn{5}{|l|}{ From COVID-19 stressors $\rightarrow$ psychological distress } \\
\hline Total effects & 0.32 & 0.03 & $<0.001$ & $0.27,0.37$ \\
\hline Direct effect & 0.27 & 0.03 & $<0.001$ & $0.22,0.31$ \\
\hline Total indirect effect & 0.05 & 0.01 & $<0.001$ & $0.03,0.07$ \\
\hline \multicolumn{5}{|l|}{ Specific indirect effects } \\
\hline COVID-19 stressors $\rightarrow$ resilience $\rightarrow$ psychological distress & 0.03 & 0.01 & 0.001 & $0.02,0.05$ \\
\hline COVI-19 stressors $\rightarrow$ institutional support $\rightarrow$ psychological distress & 0.01 & 0.004 & 0.042 & $0.002,0.01$ \\
\hline $\begin{array}{l}\text { COVID-19 stressors } \rightarrow \text { institutional support } \rightarrow \text { resilience } \rightarrow \text { psychological } \\
\text { distress }\end{array}$ & 0.01 & 0.003 & 0.002 & $0.01,0.02$ \\
\hline \multicolumn{5}{|l|}{ From institutional support $\rightarrow$ psychological distress } \\
\hline Total effects & -0.17 & 0.03 & $<0.001$ & $-0.21,-0.12$ \\
\hline Direct effect & -0.07 & 0.03 & 0.011 & $-0.12,-0.03$ \\
\hline \multicolumn{5}{|l|}{ Indirect effect } \\
\hline Institutional support $\rightarrow$ resilience $\rightarrow$ psychological distress & -0.10 & 0.01 & $<0.001$ & $-0.12,-0.08$ \\
\hline
\end{tabular}

psychological distress in HIV healthcare providers who were at risk for highly stressful experiences during the COVID-19 pandemic.

Our study found $38.7 \%$ (33.4\% mild, 5.4\% moderate, $0.3 \%$ severe) Chinese HIV healthcare providers exhibited psychological distress during the COVID-19 pandemic, which was similar to yet higher than the findings in a previous meta-analysis study (pooled prevalence of $23.2 \%$ for anxiety and $22.8 \%$ for depression) in Chinese healthcare providers [9]. Our findings indicated that during the COVID19 pandemic, HIV healthcare providers, similar to nurses and surgical staffs, were highly vulnerable to psychological distress. Their psychological health should not be neglected and merits more attention.

Our serial indirect model plotted a stress influences on psychological distress among Chinese HIV healthcare providers in response to the emergent pandemic. Results suggested that such stress influences occurred through two intermediating factors, namely institutional support (at organizational level) and resilience (at intrapersonal level). This finding provided a novel insight regarding COVID19 stress management at HIV clinics, particularly on the importance of institutional support. Previous studies showed that healthcare providers in supportive working environments were inclined to exhibit greater resilience-related abilities, including emotional regulation [47], self-efficacy [48], and adaptive coping strategies [49]. In facing a public health emergency, sufficient institutional support might enable HIV healthcare providers to use their internal coping recourses (i.e., resilience) and facilitate providers' adaptive coping instead of being overwhelmed to deal with COVID19 stressors, resulting in reduced levels of distress. This implied that psychological health among HIV healthcare providers could benefit from effective institute responses to
COVID-19, such as COVID-19 training and guidelines, sufficient supplies of personal protection equipment, and cares of workers' personal and family needs [19, 50].

In addition to institutional support, the protective and mediating role of resilience also highlighted the effects of personal coping abilities on psychological health in Chinese HIV healthcare providers during the COVID-19 pandemic. In face of the extreme pandemic stress, the availability of cognitive resources (e.g., self-efficacy) might allow HIV healthcare providers to reappraise stressors as controllable or manageable instead of threatening, resulting in reduced levels of perceived stress and a lower likelihood of emotional exhaustion [51]. The role of resilience supports the value of providing resilience-enhancing interventions for HIV healthcare providers during the COVID-19 pandemic. For example, group-based resilience-enhancing intervention programs have shown psychological benefits to healthcare providers [52]. After resilience-based cognitive trainings (e.g., selfawareness and meditation practice) at workplaces, healthcare providers demonstrated significant improvements in resilience, perceived stress, and interpersonal abilities (e.g., empathy and understanding of peers) comparing to those in control group [53, 54]. It may be worthwhile to adapt the group-based resilience-enhancing intervention and tailor it to the COVID-19 context for HIV healthcare providers in China.

There are several limitations in the current study. First, the participants were recruited online using a convenience sampling approach. Although the current study has covered 14 counties in Guangxi, our findings may not be generalized to other regions in China and may be limited by only reaching participants who are familiar with web-based devices. Future research would benefit from using a random sampling approach and recruiting participants from multiple 
Chinese regions. Second, given cross-sectional data, the indirect model from COVID-19 stressors to psychological distress cannot draw conclusion about causality. Future longitudinal research is needed to ascertain the directionality of relationships identified in this study. Third, self-report data were subject to bias (e.g., social desirability). Fourth, some measures were self-developed (COVID-19 stressors and institutional support) and future research is needed to validate these measures. Fifth, data were collected in a certain period of the COVID-19 outbreak. Our results may not be replicated as the pandemic evolves and HIV healthcare providers' experience changes. Future research would benefit from collecting data across different phases of the pandemic.

Despite these limitations, as the first attempt to examine psychological distress and its related factors among Chinese HIV healthcare providers during the COVID-19 pandemic, our study offered some important insights. First, the current study expanded the knowledge of healthcare providers' psychological health during the COVID-19 pandemic by attending to HIV healthcare providers. Psychological distress was prevalent (38.7\%) among HIV healthcare providers during the COVID-19 pandemic in China. Second, by using an ecological perspective, we identified that institutional support (as interpersonal factor) and resilience (as intrapersonal factor) mitigated the influences of COVID-19 stress on psychological distress among HIV healthcare providers in China. Our findings implied that multi-level intervention approaches, such as the establishment of supportive environment at HIV clinics and the resilience-enhancing intervention, should be utilized for promoting psychological health among HIV healthcare providers in China.

Acknowledgements We appreciated Ms. Shuaifeng Liu for her assistance in IRB application and project coordination. We also greatly thank all the HIV care providers who finished the online survey. Research reported in this publication was supported by the National Institute of Allergy and Infectious Diseases of the National Institutes of Health under Award Number R01AI127203-4S1 and the University of South Carolina Office of Vice President for Research COVID-19 grant (USCIP 80003673). The content is solely the responsibility of the authors and does not necessarily represent the official views of the National Institutes of Health. Work by Shufang Sun was also supported by National Institute of Health (T32MH078788 and K23AT011173).

\section{Compliance with Ethical Standards}

Conflict of interest The authors have no conflicts of interest to report.

\section{References}

1. World Health Organization. Novel Coronavirus (2019-NCoV) Situation Report B. https://www.who.int/docs/default-source/ coronaviruse/situation-reports/20200204-sitrep-15-ncov.pdf?sfvrs $\mathrm{n}=88 \mathrm{fe} 8$ ad6_2 (2020).
2. Bartsch SM, Ferguson MC, McKinnell JA, et al. The potential health care costs and resource use associated with COVID-19 in the United States: a simulation estimate of the direct medical costs and health care resource use associated with COVID-19 infections in the United States. Health Aff. 2020;39:10-1377.

3. Coibion O, Gorodnichenko Y, Weber M. Labor markets during the Covid-19 crisis: a preliminary view. Cambridge: National Bureau of Economic Research; 2020.

4. Kawohl W, Nordt C. COVID-19, unemployment, and suicide. Lancet Psychiatry. 2020;7(5):389-90.

5. Gunnell D, Appleby L, Arensman E, et al. Suicide risk and prevention during the COVID-19 pandemic. Lancet Psychiatry. 2020;7(6):468-71.

6. Pfefferbaum B, North CS. Mental health and the Covid-19 pandemic. N Engl J Med. 2020;383:510.

7. Sultana A, Sharma R, Hossain MM, Bhattacharya S, Purohit N. Burnout among healthcare providers during COVID-19 pandemic: challenges and evidence-based interventions (2020).

8. Wallace CL, Wladkowski SP, Gibson A, White P. Grief during the COVID-19 pandemic: considerations for palliative care providers. J Pain Symptom Manag. 2020;60:e70.

9. Pappa S, Ntella V, Giannakas T, Giannakoulis VG, Papoutsi E, Katsaounou P. Prevalence of depression, anxiety, and insomnia among healthcare workers during the COVID-19 pandemic: a systematic review and meta-analysis. Brain Behav Immun. 2020;88:901.

10. Chew NWS, Lee GKH, Tan BYQ, et al. A multinational, multicentre study on the psychological outcomes and associated physical symptoms amongst healthcare workers during COVID19 outbreak. Brain Behav Immun. 2020;88:559.

11. Huang Y, Zhao N. Generalized anxiety disorder, depressive symptoms and sleep quality during COVID-19 outbreak in China: a web-based cross-sectional survey. Psychiatry Res. 2020;288:112954.

12. Xu J, Xu Q, Wang C, Wang J. Psychological status of surgical staff during the COVID-19 outbreak. Psychiatry Res. 2020;288:112955.

13. Li Z, Ge J, Yang M, et al. Vicarious traumatization in the general public, members, and non-members of medical teams aiding in COVID-19 control. Brain Behav Immun. 2020;88:916.

14. Mgbako O, Miller EH, Santoro AF, et al. COVID-19, telemedicine, and patient empowerment in HIV care and research. AIDS Behav. 2020;24:990.

15. Sun S, Hou J, Chen Y, Lu Y, Brown L, Operario D. Challenges to HIV care and psychological health during the COVID-19 pandemic among people living with HIV in China. AIDS Behav. 2020. https://doi.org/10.1007/s10461-020-02903-4.

16. Sasangohar F, Jones SL, Masud FN, Vahidy FS, Kash BA. Provider burnout and fatigue during the COVID-19 pandemic: lessons learned from a high-volume intensive care unit. Anesth Analg. 2020;131(1):106-11. https://doi.org/10.1213/ ANE.0000000000004866.

17. Bronfenbrenner U. Ecological models of human development. Read Dev Child Int Ensyclopedia Educ. 1994;3:1643.

18. Tudge JRH, Payir A, Merçon-Vargas E, et al. Still misused after all these years? A reevaluation of the uses of Bronfenbrenner's bioecological theory of human development. J Fam Theory Rev. 2016;8(4):427-45.

19. Shanafelt T, Ripp J, Trockel M. Understanding and addressing sources of anxiety among health care professionals during the COVID-19 pandemic. JAMA. 2020;323(21):2133-4.

20. Zhang SX, Sun S, Jahanshahi AA, et al. Developing and testing a measure of COVID-19 organizational support of healthcare workers-results from Peru, Ecuador, and Bolivia. Psychiatry Res. 2020;291:113174. 
21. Dugani S, Afari H, Hirschhorn LR, et al. Prevalence and factors associated with burnout among frontline primary health care providers in low- and middle-income countries: a systematic review. Gates Open Res. 2018;2:4. https://doi.org/10.12688/gatesopenr es.12779.3.

22. Bronkhorst B, Tummers L, Steijn B, Vijverberg D. Organizational climate and employee mental health outcomes: a systematic review of studies in health care organizations. Health Care Manag Rev. 2015;40(3):254-71.

23. Thorsteinsson EB, Brown RF, Richards C. The relationship between work-stress, psychological stress and staff health and work outcomes in office workers. Psychology. 2014;5:1301.

24. Zheng J, Wu G. Work-family conflict, perceived organizational support and professional commitment: a mediation mechanism for Chinese project professionals. Int J Environ Res Public Health. 2018;15(2):344.

25. Balicer RD, Omer SB, Barnett DJ, Everly GS. Local public health workers' perceptions toward responding to an influenza pandemic. BMC Public Health. 2006;6(1):99.

26. Connor KM, Davidson JRT. Development of a new resilience scale: the Connor-Davidson Resilience scale (CD-RISC). Depress Anxiety. 2003. https://doi.org/10.1002/da.10113.

27. Luthar SS, Zigler E. Vulnerability and competence: a review of research on resilience in childhood. Am J Orthopsychiatry. 1991;61(1):6.

28. Hu T, Zhang D, Wang J. A meta-analysis of the trait resilience and mental health. Pers Individ Diff. 2015. https://doi.org/10.1016/j. paid.2014.11.039.

29. Prime H, Wade M, Browne DT. Risk and resilience in family wellbeing during the COVID-19 pandemic. Am Psychol. 2020;75:631.

30. Yan H, Li X, Li J, et al. Association between perceived HIV stigma, social support, resilience, self-esteem, and depressive symptoms among HIV-positive men who have sex with men (MSM) in Nanjing, China. AIDS Care. 2019;31(9):1069-76.

31. Gao M, Xiao C, Zhang X, Li S, Yan H. Social capital and PTSD among PLWHA in China: the mediating role of resilience and internalized stigma. Psychol Health Med. 2018;23(6):698-706.

32. Cai W, Lian B, Song X, Hou T, Deng G, Li H. A cross-sectional study on mental health among health care workers during the outbreak of Corona Virus Disease 2019. Asian J Psychiatry. 2020;51:102111.

33. Liu L, Hu S, Wang L, Sui G, Ma L. Positive resources for combating depressive symptoms among Chinese male correctional officers: perceived organizational support and psychological capital. BMC Psychiatry. 2013;13(1):89.

34. Sun X, Yang W, Tang S, et al. Declining trend in HIV new infections in Guangxi, China: insights from linking reported HIV/ AIDS cases with CD4-at-diagnosis data. BMC Public Health. 2020;20(1):1-12.

35. Dong L, Bouey J. Public mental health crisis during COVID-19 pandemic, China. Emerg Infect Dis. 2020;26(7):1616.

36. Chinese Center for Disease Control and Prevention. Up-to-day estimates of the COVID-19 outbreak in China. https://2019ncov. chinacdc.cn/2019-nCoV/. (2020).

37. Wang Y, Yang YY, Li S, Lei XM, Yang YF. Investigation on the status of influencing factors for depression symptom of children and adolescents with home quarantine during the prevalence of novel coronavirus pneumonia. Chin J Child Health Care. 2020;03(02):18.
38. Kroenke K, Spitzer RL, Williams JBW, Löwe B. An ultra-brief screening scale for anxiety and depression: the PHQ-4. Psychosomatics. 2009;50(6):613-21.

39. Wang W, Bian Q, Zhao Y, et al. Reliability and validity of the Chinese version of the Patient Health Questionnaire (PHQ-9) in the general population. Gen Hosp Psychiatry. 2014;36(5):539-44.

40. Campbell-Sills L, Stein MB. Psychometric analysis and refinement of the connor-davidson resilience scale (CD-RISC): validation of a 10-item measure of resilience. J Trauma Stress. 2007;20(6):1019-28.

41. Meyers LS, Gamst G, Guarino AJ. Applied multivariate research: design and interpretation. Thousand Oaks: Sage publications; 2016.

42. Kline RB. Principles and practice of structural equation modeling. New York: Guilford; 1998.

43. Kim H-Y. Statistical notes for clinical researchers: assessing normal distribution (2) using skewness and kurtosis. Restor Dent Endod. 2013;38(1):52-4.

44. Hu L, Bentler PM. Cutoff criteria for fit indexes in covariance structure analysis: conventional criteria versus new alternatives. Struct Equ Model. 1999;6(1):1-55.

45. Muthén L, Muthén B. Mplus user's guide. 8th ed. Los Angeles: Muthén \& Muthén; 2017.

46. Baron RM, Kenny DA. The moderator-mediator variable distinction in social psychological research: conceptual, strategic, and statistical considerations. J Pers Soc Psychol. 1986;51(6):1173.

47. Chang CJ, Fehling KB, Selby EA. Sexual minority status and psychological risk for suicide attempt: a serial multiple mediation model of social support and emotion regulation. Front Psychiatry. 2020. https://doi.org/10.3389/fpsyt.2020.00385.

48. Shoji K, Bock J, Cieslak R, Zukowska K, Luszczynska A, Benight CC. Cultivating secondary traumatic growth among healthcare workers: the role of social support and self-efficacy. J Clin Psychol. 2014;70(9):831-46.

49. Franz S, Zeh A, Schablon A, Kuhnert S, Nienhaus A. Aggression and violence against health care workers in Germany-a cross sectional retrospective survey. BMC Health Serv Res. 2010;10(1):51.

50. Morgantini LA, Naha U, Wang H, et al. Factors contributing to healthcare professional burnout during the COVID-19 pandemic: a rapid turnaround global survey. medRxiv. 2020. https://doi. org/10.1101/2020.05.17.20101915.

51. Cohen F, Lazarus RS. Coping and adaptation in health and illness. In: Rosmarin DH, Koenig HG, editors. Handbook health, health care, health professional. Amsterdam: Elsevier; 1983. p. 608-35.

52. Scheepers RA, Emke H, Epstein RM, Lombarts KM. The impact of mindfulness-based interventions on doctors' well-being and performance: a systematic review. Med Educ. 2020;54(2):138-49.

53. Verweij H, van Ravesteijn H, van Hooff MLM, Lagro-Janssen ALM, Speckens AEM. Mindfulness-based stress reduction for residents: a randomized controlled trial. J Gen Intern Med. 2018;33(4):429-36.

54. Sood A, Prasad K, Schroeder D, Varkey P. Stress management and resilience training among Department of Medicine faculty: a pilot randomized clinical trial. J Gen Intern Med. 2011;26(8):858-61.

Publisher's Note Springer Nature remains neutral with regard to jurisdictional claims in published maps and institutional affiliations. 